\title{
Critical Currents of Bismuth 1G Tape
}

\author{
W.M. Woch*, R. Zalecki, M. Chrobak And A. KolodziejczyK \\ AGH University of Science and Technology, Faculty of Physics and Applied Computer Science, \\ Solid State Physics Department, Cracow, Poland
}

\begin{abstract}
The critical currents of commercial bismuth based superconducting tape were determined in the two ways. In the first one the transport critical current density was measured by the four points method using the dc current power supply at the liquid nitrogen temperature. In the second one the critical current densities were obtained from the absorption part of ac susceptibility measurements using the Bean model near the critical temperature. The temperature dependence of the critical current densities was fitted to take advantage of the Ginzburg-Landau strong-coupling limit approach. Using the fit parameters the critical current density at $77 \mathrm{~K}$ was calculated. The critical temperature of this tape $\left(T_{\mathrm{c}}=110.5 \mathrm{~K}\right)$ was determined from the ac susceptibility measurements.
\end{abstract}

DOI: $10.12693 /$ APhysPolA.127.315

PACS: 74.72.-h, 74.25.F-, 74.25.Ha, 74.25.Sv

\section{Introduction}

The preparation of high temperature superconductors (HTS) tapes and films has gained much interest for largescale applications including power system components such as electrical motors, generators, power transmission cable, transformers, and superconducting magnetic energy storage devices $[1,2]$. Extensive investigations have been connected to optimizing the fabrication and processing techniques of HTS wires and tapes [3]. Typical critical current densities of tapes are of the order of $30-35 \mathrm{kAcm}^{-2}$ at $77 \mathrm{~K}$ and the self magnetic field that gives the critical currents of the order of 120-170 A [4, 5]. These critical currents decrease to $20-60 \mathrm{~A}$ in the magnetic field $B=1 \mathrm{~T}$ at $77 \mathrm{~K}$. One of the highest reported critical current densities was about $110 \mathrm{kAcm}^{-2}$ at $77 \mathrm{~K}[6]$.

In this paper the results of ac susceptibility as well as the transport critical current measurements of the first generation $(1 \mathrm{G})$ bismuth based superconducting tape are presented. The temperature dependences of the absorption part of ac susceptibility were carried out to evaluate the critical current densities using the Bean critical state model.

\section{Experimental}

The commercial first generation bismuth based superconducting tape was manufactured by the American Superconductor Company (AMSC). The commercially identifying of this tape is as follows: 1G High Strength Plus 135A Ic min "5715 135" BSCCO.

The transport critical current density was measured by the four points method using the dc current power supply at the liquid nitrogen temperature. For these measurements the $\mathrm{H}$-like shape of the sample was prepared.

\footnotetext{
* corresponding author; e-mail: wmwoch@agh.edu.pl
}

The cross-section of the tape is as follows: $0.1 \times 0.012 \mathrm{~cm}^{2}$. The gap between the voltage contacts is $3 \mathrm{~mm}$. The dispersion $\chi^{\prime}$ and absorption $\chi^{\prime \prime}$ parts of the ac susceptibility as a function of temperature in magnetic field amplitudes from 0.2 Oe up to 28 Oe were measured by a standard mutual inductance bridge operating at the frequency of $19 \mathrm{~Hz}$. A Stanford SR 830 lock-in nanovoltmeter served both as a source for the ac current for the coil which produced the ac magnetic field and as a voltmeter of the bridge. The temperature was monitored by a Lake Shore temperature controller employing a chromelgold- $0.07 \%$ Fe thermocouple with an accuracy of $\pm 0.05 \mathrm{~K}$ for this experimental setup. The magnetic field was parallel to the sample surface and the average width of the superconducting material was about $0.12 \mathrm{~mm}$.

\section{Results}

The transport critical current density of the first generation bismuth based tape was measured by the four points technique using the dc current power supply. This measurements have been done at the liquid nitrogen temperature. The voltage drop as a function of applied dc

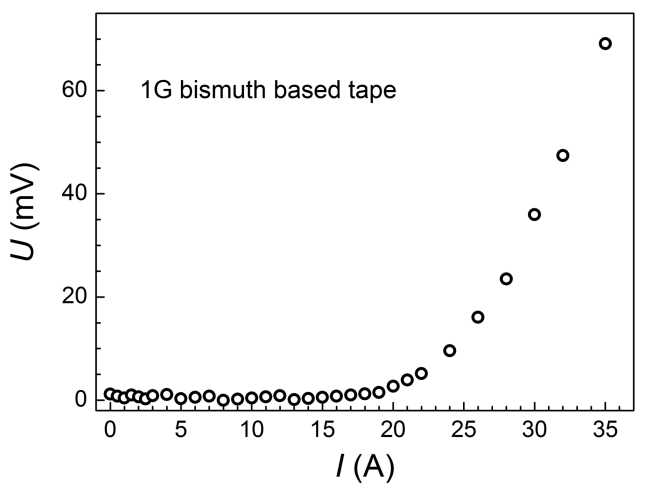

Fig. 1. Voltage drop as a function of applied dc currents in the liquid nitrogen temperature. The critical current density was obtained to be $J_{\mathrm{c}}=17.5 \mathrm{Acm}^{-2}$ at $77 \mathrm{~K}$ in the self magnetic field. 
currents is shown in Fig. 1. According to this dependence one can notice that at the current $I=21$ A the voltage drop starts to increase. Then this value of the current was taken to calculate the critical current density. Using the cross-section $S=0.0012 \mathrm{~cm}^{2}$ the critical current density was obtained to be $J_{\mathrm{c}}=17.5 \times 10^{3} \mathrm{Acm}^{-2}$ at $77 \mathrm{~K}$ in the self magnetic field. The transport critical current flows through the grains as well as through the inter-grain links which may be treated as the Josephson junctions. The critical current is limited by the weakest junctions on the current percolative path.

The absorption as well as the dispersion parts ac susceptibility as a function of temperature of the BSCCO tape are shown in Fig. 2a and b, respectively. The bismuth based tape shows very good quality of the superconducting material with the onset of the critical temperature $T_{\mathrm{c}}=110.5 \mathrm{~K}$ and narrow transition to the superconductivity state. The absorption part of the ac susceptibility exhibits in general one peak concerning to the energy losses of the inter-granular regions because these peaks move to the lower temperatures when the applied magnetic field is being increased. However, even in the highest ac field amplitudes over 25 Oe the peak position is still over $100 \mathrm{~K}$ (see Fig. 2a) so it means that the critical current densities in the sample are very high.

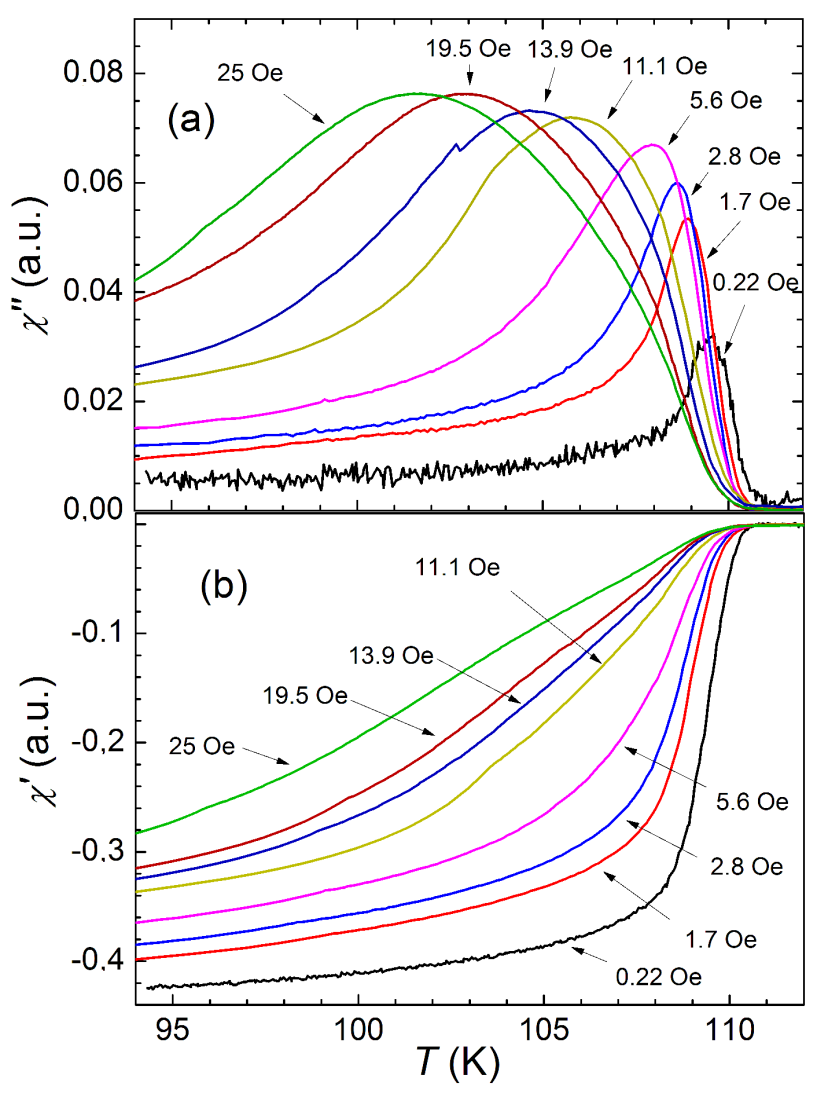

Fig. 2. Absorption (a) as well as the dispersion (b) parts ac susceptibility as a function of temperature of the BSCCO tape at the different ac magnetic fields indicated by arrows.
To obtain the critical current densities from the peak position of the absorption part of susceptibility the Bean critical state model was implemented. We derived the $J_{\mathrm{c}}$ values from the formula $[7,8]$ :

$$
J_{\mathrm{c}}=\frac{2 H_{\mathrm{ac}}}{d},
$$

where $H_{\mathrm{ac}}$ is the ac magnetic field amplitude and $d$ is the sample thickness in the perpendicular direction to the ac field. These critical current densities are linked to the inter-grain properties of the superconducting material. The results of the inter-grain critical current density calculations for the BSCCO tape according to Eq. (1) are shown in Fig. 3. After the Ginzburg-Landau strong coupling limit approach the critical current varies with temperature according to the equation [9]:

$$
J_{\mathrm{c}}=J_{\mathrm{c} 0}\left(1-\frac{T}{T_{\mathrm{c}}}\right)^{n},
$$

where $T_{\mathrm{c}}$ is the critical temperature that depends on the applied magnetic field and $J_{c 0}$ is the critical current at $0 \mathrm{~K}$. Originally the exponent $n$ was determined to be 1.5 [10] but it may vary within a wide range [11]. If the exponent $n$ is greater than unity there is strong pinning and a vortex glass structure can be observed. In this case, the function expressed by Eq. (2) has a slightly upward curvature which is typical for HTS. If the exponent $n$ is less than unity the pinning force is rather weak and limits the critical current. Then the function expressed by Eq. (2) has a downward curvature typical for low temperature superconductors.

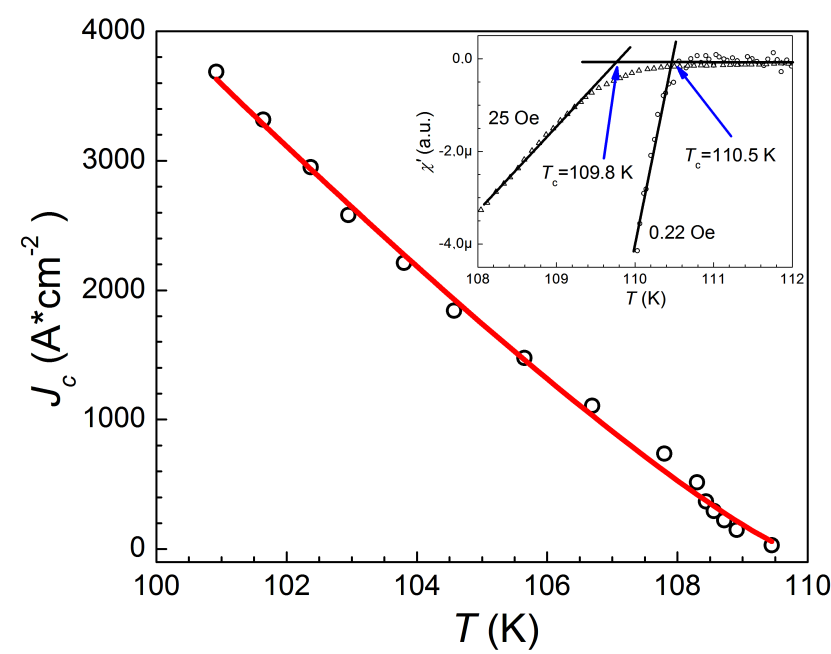

Fig. 3. Critical current as a function of temperature calculated from the Bean critical state model (open circles). The solid line is the fit Eq. (2). Inset: the manner of the determination of the onset of the critical temperature.

The temperature dependence of the critical current density of the tape was fitted using Eq. (2) in which the critical temperature $-T_{\mathrm{c}}$, the critical current at $0 \mathrm{~K}-$ $J_{\mathrm{c} 0}$ and the exponent $n$ are the fit parameters. We assumed that the critical temperature does not depend on the applied magnetic field in this experiment because 
it varies from $T_{\mathrm{c}}=110.5 \mathrm{~K}$ for 0.22 Oe to $T_{\mathrm{c}}=109.8 \mathrm{~K}$ for 25 Oe (see inset in Fig. 3). The solid line that is shown in Fig. 3 presents fit of Eq. (2) to the experimental data. The fit parameters: $T_{\mathrm{c}}$ and $n$ as well as the critical current density in the liquid nitrogen temperature $J_{\mathrm{c}}(77 \mathrm{~K})$, calculated according to the formula (2) are as follows: $T_{\mathrm{c}}=109.7 \pm 0.2 \mathrm{~K}, n=1.18 \pm 0.07$ and $J_{\mathrm{c}}(77 \mathrm{~K})=16.9 \mathrm{kAcm}^{-2}$. This critical temperature is almost the same as the zero critical temperature $T_{\mathrm{c} 0}=109.8 \mathrm{~K}$ obtained from the temperature dependence of resistivity measurements without the applied magnetic field. Comparison of the critical current densities at $77 \mathrm{~K}$ obtained from the transport as well as the ac susceptibility measurements show that these values are almost the same. One can say that the ac susceptibility measurements are alternative method to get the transport critical currents. The exponent $n=1.18$ higher than unity is typical for HTS that exhibit upward curvature of $J(T)$ dependences. It means that there is the vortex glass structure with the pinning force which plays important role especially at the lower temperatures.

\section{Summary}

The measured commercial first generation bismuth based tape has very good superconducting quality such as high value of critical temperature of $110 \mathrm{~K}$ and the narrow transition width to the superconducting state. The critical current densities at $77 \mathrm{~K}$ obtained from the transport measurements is found to be $J_{\mathrm{c}}=17.5 \times$ $10^{3} \mathrm{Acm}^{-2}$ while obtained from the ac susceptibility measurements using the Bean model is found to be $J_{\mathrm{c}}=$ $16.9 \times 10^{3} \mathrm{Acm}^{-2}$. These values are almost the same. The exponent $n=1.18$ higher than unity is typical for HTS that exhibit upward curvature of $J(T)$ dependences. It means that there is the vortex glass structure with the pinning force which plays important role especially at the lower temperatures.

These parameters prove that BSCCO is the good superconducting material for the applications.

\section{Acknowledgments}

This work was supported by the Polish Ministry of Science and Higher Education and its grants for Scientific Research. One of us (M.Ch.) has been partly supported by the EU Human Capital Operation Program, Polish Project No. POKL.04.0101-00-434/08-00.

\section{References}

[1] H.J.M. ter Brake, F.-Im. Buchholz, G. Burnell, T. Claeson, D. Crété, P. Febvre, G.J. Gerritsma, H. Hilgenkamp, R. Humphreys, Z. Ivanov, W. Jutzi, M.I. Khabipov, J. Mannhart, H.-G. Meyer, J. Niemeyer, A. Ravex, H. Rogalla, M. Russo, J. Satchell, M. Siegel, H. Töpfer, F.H. Uhlmann, J.-C. Villégier, E. Wikborg, D. Winkler, A.B. Zorin, Physica C 439, 1 (2006).

[2] N. Ayai, M. Ueyama, T. Kato, S. Kobayashi, A. Mikumo, T. Kaneko, T. Hikata, K. Hayashi, H. Takei, Advances in Superconductivity XII, Springer-Verlag, Tokyo 2000, p. 631.

[3] See for instance: J. Azman, H. Abdullah, R. AbdShukor, Adv. Condens. Matter Phys. 2014, 1 (2014), and references therein.

[4] P. Sunwong, J.S. Higgins, D.P. Hampshire, IEEE Trans. Appl. Superconduct. 21, 2840 (2011), and references therein.

[5] G. Grassot, A. Jeremie, R. Flukiger, Supercond. Sci. Technol. 8, 827 (1995), and references therein.

[6] M. Lelovic, P. Krishnaraj, N.G. Eror, U. Balachandran, Physica C 242, 246 (1995).

[7] C.P. Bean Phys. Rev. Lett. 8, 250 (1962).

[8] J.R. Clem, Physica C 153-155, 50 (1988).

[9] J.R. Clem, B. Bumble, S.I. Raider, W.J. Gallagher, Y.C. Shih, Phys. Rev. B 35, 7526 (1987).

[10] K.A. Müller, M. Takashige, J. Bednorz, Phys. Rev. Lett. 58, 1143 (1987); Y. Yeshurun, A.P. Malozemoff, Phys. Rev. Lett. 60, 2202 (1988).

[11] W.M. Woch, R. Zalecki, A. Kołodziejczyk, H. Sudra, G. Gritzner, Supercond. Sci. Technol. 21, 085002 (2008). 